02

\title{
О выборе волновой функции основного состояния Не для прецизионных расчетов параметров автоионизационных состояний выше порога образования возбужденных ионов
}

\author{
(C) Т.М. Заяц, ${ }^{1}$ B.М. Симулик, ${ }^{2, \text { Ф Р.В. Тимчик }}{ }^{2}$ \\ ${ }^{1}$ Ужгородский национальный университет, \\ 88000 Ужгород, Украина \\ ${ }^{2}$ Институт электронной физики НАН Украины, \\ 88000 Ужгород, Украина \\ T e-mail: vsimulik@gmail.com
}

(Поступило в Редакцию 22 сентября 2017 г.)

Необходимость выбора многопараметрических волновых функций для описания основного состояния атома в задачах ионизации атомов фотонами и электронами обоснована на примере атома Не. Выполнен сравнительный анализ применения различных волновых функций основного состояния этого атома. Вычислены энергии, ширины и парциальные ширины нижнего ${ }^{1} P$ автоионизационного состояния Не, находящегося выше порога образования возбужденных ионов. Показано, что в отличие от полных ширин квазистационарных состояний, меняющихся для разных волновых функций основного состояния незначительно, парциальные ширины при этом существенно отличаются друг от друга.

DOI: 10.21883/JTF.2018.07.46161.2491

\section{Введение}

В современных расчетах сечений ионизации атомов фотонами, электронами или другими частицами волновая функция начального состояния, как правило, выбирается в том же приближении, что и волновая функция конечного состояния. Это показано, например, в работах Берка [1], Берка, Беррингтона, Сукумара [2] и Люка [3].

Фано в [4] и Фано, Купер в [5] аргументированно доказали, что при анализе процессов возбуждения двухчастичных состояний у большого количества атомов химических элементов в основном состоянии в первую очередь необходимо учитывать многоэлектронные корреляции типа $(n l)^{2}$. Это связано с тем фактом, что при наличии всего двух электронов на единственной в атоме оболочке важными являются корреляции в основном состоянии.

В случае расчетов сечений ионизации двухэлектронных систем, например атома гелия выше порога образования возбужденных ионов, а именно $\mathrm{He}^{+}(N=2)$, этот вопрос имеет принципиальное значение, поскольку наряду с одночастичным каналом $1 s \varepsilon l$ в этой задаче следует учитывать связь с двукратно возбужденными каналами $2 s \varepsilon L, 2 p \varepsilon(L-1), 2 p \varepsilon(L+1)$. Амплитуда возбуждения этих каналов обусловлена как их связью с каналом $1 s \varepsilon l$ в конечном состоянии, так и многоэлектронными корреляциями в основном состоянии.

Задача расчета волновой функции основного состояния рассматриваемой здесь модельной системы, которая описывается проекцией гамильтониана на подпространство $1 s-, 2 s-, 2 p$-состояний иона $\mathrm{He}^{+}$, соответствует решению задачи на связь трех закрытых каналов. Система уравнений координатного представления метода сильной связи каналов в этом случае превращается в уравнения Хартри-Фока в многоконфигурационном подходе [6-9].

Решение этой системы уравнений методом взаимодействующих конфигураций соответствует проблеме нахождения собственных значений матрицы вещественного симметричного оператора бесконечного ранга. Касательно двухэлектронных систем это соответствует решению многоконфигурационной проблемы с учетом $(n l)^{2}$ конфигураций соответствующего атома.

\section{Задача о выборе волновой функции основного состояния атома гелия}

Выбор волновой функции основного состояния атома - это важный шаг в теоретическом описании ионизации атомов. Критерием выбора той или иной волновой функции основного состояния является значение энергии основного состояния атома, которое получается в расчетах на основе использования той или иной волновой функции. Рассчитанное значение энергии основного состояния не всегда совпадает с экспериментальным значением энергии. А в случае прецизионных расчетов параметров автоионизационных состояний (АИС) этот факт будет иметь принципиальное значение. Поэтому для таких расчетов выбирают волновую функцию, которая точно отображает экспериментальное значение энергии основного состояния. Наиболее известным классом функций основного состояния гелиеподобных систем является класс волновых функций, представленных 
в работах Хиллерааса [10-12]:

$$
\left(\Psi_{0}=\sum_{n, l, m} C_{n l m} \psi_{n l m}, l=0,2,4, \ldots\right)
$$

где $\psi_{n l m}=s^{n} t^{l} w^{m} \exp (-s / 2) /(n+l+m+2)$ !. Здесь $s=$ $=r_{1}+r_{2}, t=r_{2}-r_{1}, u=r_{12}-$ независимые переменные, определяющие вариационную задачу, а $r_{1}, r_{2}, r_{3}-$ это привычные обозначения взаимных расстояний обоих электронов и ядра, $n, l, m-$ квантовые числа (главное, орбитальное и магнитное соответственно), $C_{n l m}$ - соответствующие коэффициенты разложения по собственным функциям $\psi_{n l m}$ (см. также, например, формулы (10), (10a) в [12] и комментарии к ним).

Эти функции хорошо описывают энергию основного состояния Не, однако их применение для задач ионизаци в большой мере усложняется методикой расчета амплитуд, сложность расчета которых обусловлена в основном нахождением интегралов, в которые входит множитель вида $\left|r_{1}-r_{2}\right|^{m}$. В случае использования методов сильной связи каналов в координатном представлении расчет амплитуд выполняется один раз в каждой точке по энергии.

Подробный анализ расчета параметров по методике Хиллерааса представлен в работе [13] с учетом модификации Фока [14]. В работе [13] показано, что уже в шестом приближении:

$$
\Psi^{(6)}=\Psi^{(4)}+\left[c_{5} u^{5} r^{-2}+c_{6}\left(5 s+t^{2} / s\right) r^{3}\right] \exp (-z S),
$$

где

$$
\begin{aligned}
\Psi^{(4)}= & \exp (-z S)\left\{1+u / 2+(1+u / 2) R_{1}\right. \\
& \left.\times\left[c_{1} r^{-1}+c_{2}+c_{3} r\right]+c_{4} u^{2} R_{1} r^{-3}\right\} .
\end{aligned}
$$

Такая функция основного состояния дает значение энергии, близкое к экспериментальному, а именно $E=2.903557$ a.e. В расчетах используются, как правило, многопараметрические волновые функции Хиллерааса (6-, 8- или даже 56-параметрические функции).

В отличие от такого типа вариационных функций волновые функции, полученные в многоконфигурационном приближении Хартри-Фока, не дают значения энергии основного состояния гелия, близкого к экспериментальному (см., например, $[8,9,15])$. Наиболее точным анализом многопараметрических вариационных волновых функций для атома гелия считаем даный в работах Пекериса $[16,17]$. Однако использование таких функций в серийных расчетах является сложным и громоздким.

Решение уравнения Шредингера для основного состояния Не удобнее искать в классе функций с переменными, которые разделяются. Наиболее известными среди них является аналитическая форма волновой функции Хартри-Фока в одноконфигурационном приближении

$$
\chi(r)=N_{r}(\exp (-\xi r)+0.6 \exp (-\xi r))
$$

и корреляционная функция Эккарта

$$
\chi(\alpha r)=N_{a}(\exp (-\alpha r)), \quad \alpha=2 / a_{0},
$$

где $a_{0}$ - радиус Бора, а буквами $\xi$ и $\xi$ в (3) обозначены числовые параметры, которые объяснены, например, в $[18,19]$. Коэффициенты $N_{r}$ и $N_{a}$ в этих функциях вибираются из условия нормировки волновой функции $\Psi_{0}\left(r_{1}, r_{2}\right)$ на единицу. Однако такого вида функции используются только для грубых оценок.

Согласно [20], волновая функция основного состояния записывается следующим образом:

$$
\Psi_{0}\left(r_{1} r_{2}\right)=\sum_{l} B_{l} Y_{l}^{00}\left(r_{12}\right) F_{l}\left(r_{1} r_{2}\right)
$$

где $Y_{l}^{00}$ может быть представлена в виде $Y_{l}^{00}=$ $=\sum_{\mu} Y_{l \mu}\left(\theta_{1}, \varphi_{1}\right) C_{l \mu l-\mu}^{00} Y_{l \mu}\left(\theta_{2}, \varphi_{2}\right), \quad B_{l}-$ коэффициент разложения, $r_{i}$ - радиус-вектор $i$-го электрона, $r_{i}-$ соответствующий модуль, $C_{l \mu l-\mu}^{L M} \equiv\langle l \mu l-\mu \mid L M\rangle-$ коэффициенты векторного сложения, $F_{l}\left(r_{1}, r_{2}\right)$ - радиальные функции, описывающие вклад $(n l)^{2}$ конфигураций в основное состояние. Выбор пробних функций $F_{l}\left(r_{1}, r_{2}\right)$ в общем случае произвольный. Требуется только линейная независимость набора и обеспечение правильной асимптотики решения. В таком случае функции $F_{l}\left(r_{1}, r_{2}\right)$ могут быть записаны следующим образом:

$$
F_{l}\left(r_{1}, r_{2}\right)=\sum_{m n} A_{m n}^{l}\left\lfloor\chi_{m}^{\alpha}\left(r_{1}\right) \chi_{n}^{\beta}\left(r_{2}\right)+\chi_{n}^{\alpha}\left(r_{2}\right) \chi_{m}^{\beta}\left(r_{1}\right)\right\rfloor,
$$

где $\chi_{n}^{\alpha}-$ произвольные наборы непрерывных функций одной переменной; $\alpha, \beta$ - параметры, которые выбираются из вариационного принципа.

Вообще говоря, вариационный принцип при расчете функций $F_{l}\left(r_{1}, r_{2}\right)$ можно и не привлекать, а функции $\chi_{n}^{\alpha}$ выбирать в виде кулоновского базиса. Однако в этом случае разложение типа (5), (6) сходится очень медленно и для достижения нужной точности следует учитывать очень большое количество членов разложения. А применение вариационного принципа для выбора параметров $\alpha, \beta$ весьма ускоряет сходимость разложения (6). Твид в [20] сформулировал однопараметрическую вариационную задачу для рассматриваемых разложений. Он предложил искать функции $\chi_{n}^{\alpha}(r)$ в следующем виде:

$$
\chi_{n}^{\alpha}(r)=r^{n} \exp \left(-\frac{\alpha}{2} r\right) ; \quad \alpha=\beta,
$$

где $\alpha$ определяется из условия минимума энергии основного состояния. Коэффициенты $A_{n m}^{l}$ вычисляются диагонализацией матрицы соответствующего гамильтониана. В зависимости от чисел, учитываемых в разложении (6) мультиплетов, в класс волновых функций Твида входят 21-, 31- и 41-параметрические функции, содержащие соответственно $(n p)^{2},(n d)^{2}$ и $(n f)^{2}$-конфигурации. Для процедуры вычислений используем следующую формулу:

$$
F_{l}\left(r_{1}, r_{2}\right)=\sum_{m, n} A_{l m n}\left(r_{1}^{n} r_{2}^{m}+r_{1}^{m} r_{2}^{n}\right) \exp \left\{-\frac{1}{2} k\left(r_{1}+r_{2}\right)\right\} \text {. }
$$


Для завершения краткого обзора отметим, что в существенно ином подходе анализ волновых функций основного состояния двухэлектронных систем проводят при помощи метода Монте-Карло. В работе [21] это делается, например, для функции вида

$$
\begin{aligned}
& \Psi_{49}=\left(1+P_{12}\right) \exp \left(\frac{\sum_{k=0} a_{k} r_{1}^{n} r_{2}^{l} r_{12}^{m}}{\sum_{k=0} b_{k} r_{1}^{n} r_{2}^{l} r_{12}^{m}}\right. \\
& \left.+c\left(r_{1}^{2}+r_{2}^{2}-r_{12}^{2}\right) \ln \left[r_{1}^{2}+r_{2}^{2}\right]-\alpha r_{1}-\beta r_{2}\right)
\end{aligned}
$$

В этом приближении получено наиболее близкое к экспериментальному значение энергии основного состояния гелия.

Ниже используем волновую функцию (8).

\section{Расчет парциальных спектроскопических характеристик автоионизационных состояний в области между вторым и третьим порогами атома Не}

Диагонализационное приближение [22,23] дает возможность отследить, в какие именно каналы и в каком соотношении происходит распад АИС, находящихся в области выше порога образования возбужденных ионов $\mathrm{He}^{+}$. Этот вопрос особенно актуален, поскольку дает возможность обнаружить резонансы, распадающиеся фактически в какой-нибудь один канал (и в этом случае для таких состояний связью каналов можно пренебречь), а также резонансы, распадающиеся в несколько каналов, для которых учет связи каналов является обязательным. В принципе эту информацию можно получить из анализа данных путем сравнения значений положений и ширин резонансов в разных приближениях. Однако без знания парциальных ширин распада эта информация будет однобокой.

Для расчета силы осциллятора перехода (или сечения ионизации) необходимо определить амплитуду ионизации, которая в общем случае представляется в виде

$$
T_{|0\rangle \rightarrow|\lambda E\rangle}=\sqrt{C(E)}\left\langle\Psi_{\lambda}^{E(-)}|\hat{t}| 0\right\rangle,
$$

где $|0\rangle \equiv\left|n_{0} L_{0} S_{0}\right\rangle$ - волновая функция начального состояния атома, $C(E)$ - кинематический множитель.

Пусть волновые функции $|\lambda E\rangle$ в (10) удовлетворяют асимптотическим условиям $[1,2]$. Как было показано в работах [24,25], в этом случае матрица собственных состояний оператора энергии системы $A_{i \lambda}=\delta_{i \lambda}$. Тогда волновую функцию $\Psi_{\lambda}^{E(-)}\left(r_{1}, r_{2}\right)$ в (10) можем предста- вить следующим образом:

$$
\begin{aligned}
\left|\Psi_{\lambda}^{E(-)}\left(\mathbf{r}_{1} \mathbf{r}_{2}\right)\right\rangle= & |\lambda E\rangle+\sum_{m} \frac{\tilde{V}_{m \lambda}(E)}{\left.E-E_{m}(E)+i \Gamma_{m}(E) / 2\right)} \\
& \times\left(\left|\tilde{\Phi}_{m}^{E}\right\rangle-i\left|\chi_{m}^{E}\right\rangle\right),
\end{aligned}
$$

где

$$
\begin{gathered}
\left|\tilde{\Phi}_{m}^{E}\right\rangle=\left|\varphi_{m}^{c}\right\rangle+\frac{1}{\pi} \int_{0}^{\infty} \frac{\left|\chi_{m}^{E^{\prime}}\right\rangle}{E-E^{\prime}} d E^{\prime}, \\
\left|\chi_{m}^{E^{\prime}}\right\rangle=\pi \sum_{\lambda} \tilde{V}_{m \lambda}(E)|\lambda E\rangle,
\end{gathered}
$$

а индексу $\lambda$ ставим в соответствие набор квантовых чисел, который определяется соотношением для асимптотики волновых функций [1].

Подстановка выражений (11), (12) в (10) определяет парциальные амплитуды резонансной ионизации

$$
T_{|0\rangle \rightarrow|\lambda E\rangle}=t_{\lambda}^{d i r}(E)+\sum_{m} \frac{H_{m \lambda}(E)}{\varepsilon_{m}(E)+1} .
$$

В $(13) \varepsilon_{m}(E)=2\left[E-E_{m}\left(\tilde{E}_{m}\right)\right] / \Gamma_{m}\left(\tilde{E}_{m}\right)$, а $\Gamma_{m}\left(\tilde{E}_{m}\right)-$ это ширины АИС. Этим переходом мы разделяем амплитуду на два слагаемые: первое слагаемое фиксирует вклад прямого процесса, а второе - резонансного. Остальные величины, входящие в формулу (13), определяются следующими соотношениями:

$$
\begin{gathered}
t_{\lambda}^{d i r}(E)=\sqrt{C(E)}\langle\lambda E|\hat{t}| 0\rangle, \\
H_{m \lambda}(E)=2 \tilde{V}_{m \lambda}(E)\left[t_{m}(E)-i \tau_{m}(E)\right] \Gamma_{m}^{-1}(E), \\
t_{m}(E)=\sqrt{C(E)}\left\langle\tilde{\Phi}_{m}^{E}|\hat{t}| 0\right\rangle, \quad \tau_{m}(E)=\sqrt{C(E)}\left\langle\chi_{m}^{E}|\hat{t}| 0\right\rangle .
\end{gathered}
$$

Парциальная дифференциальная сила осциллятора перехода в канал ионизации $\lambda$ пропорциональна квадрату модуля выражения (13). Расчет полного сечения ионизации осуществляется суммированием всех парциальных вкладов по индексу $\lambda$. Тогда выражение для сечения параметризуется следующим образом:

$$
\sigma_{\lambda}(E)=\sigma_{\lambda}^{d i r}(E)+\sum_{m} \frac{\Gamma_{m}(E) P_{m \lambda}(E)+\varepsilon_{m}(E) Q_{m \lambda}(E)}{\varepsilon_{m}^{2}(E)+1} .
$$

Вещественные функции полной энергии $P_{m \lambda}(E)$ и $Q_{m \lambda}(E)$ являются удвоенными частями соответственно вещественной и комплексной частей комплексной функции $N_{m \lambda}(E)$, где последняя имеет вид

$N_{\alpha m}(E)=\sum_{\lambda c \alpha} H_{m \lambda}(E)\left(t_{\lambda}^{d i r}(E)+\sum_{n} \frac{H_{m \lambda}(E)}{\varepsilon_{n}(E)-\varepsilon_{m}(E)+2 i}\right)^{*}$.

Таким образом, сечение резонансной ионизации может определяться набором функций полной энергии: $\sigma_{\lambda}^{d i r}(E)$, $N_{\alpha m}(E), \varepsilon_{m}(E), \Gamma_{m}(E)$. 
Исходя из вышесказанного можно перейти к системе характеристик взаимодействующих АИС, аналогичных к введенным Фано в работе [4] для одного изолированого резонанса посредством функций $P_{m \alpha}(E), Q_{m \alpha}(E)$. Тогда параметры приобретают следующий вид:

$$
\begin{gathered}
\rho_{m \alpha}^{2( \pm)}(E)=\frac{P_{m \alpha}(E) \pm \sqrt{P_{m \alpha}^{2}(E)+Q_{m \alpha}^{2}(E)}}{\sigma_{\alpha}^{\text {dir }}(E)}, \\
q_{m \alpha}^{( \pm)}(E)=\frac{Q_{m \alpha}(E)}{P_{m \alpha}(E) \pm \sqrt{P_{m \alpha}^{2}(E)+Q_{m \alpha}^{2}(E)}} .
\end{gathered}
$$

В случае изолированного резонанса обе системы функций $q_{m \alpha}^{( \pm)}(E)$ и $\rho_{m \alpha}^{2( \pm)}(E)$ имеют простой геометрический смысл. Величины $q_{m \alpha}^{( \pm)}(E)$ определяют расстояние между экстремумами резонансной кривой и положением резонанса $E_{m}\left(\tilde{E}_{m}\right)$. А функции $\rho_{m \alpha}^{2( \pm)}(E)$ определены относительно фона амплитуды экстремумов. При наличии нескольких взаимодействующих АИС такая интерпретация функций (17) будет приближенной. Однозначно набор функций (17) в теории Фано определяется из условия $\rho^{2}>0$, что в принятых здесь обозначениях соответствует выбору знака $(+)$. Выражение для расчета сечений, таким образом, будет иметь следующий вид:

$$
\begin{aligned}
& \sigma_{\alpha}(E)=\sigma_{\alpha}^{d i r}(E) \\
& \times\left[1+\sum_{m}\left(\frac{\left[\varepsilon_{m}(E)+q_{m \alpha}(E)\right]^{2}}{\varepsilon_{m}^{2}(E)+1} \rho_{m \alpha}^{2}(E)-\rho_{m \alpha}^{2}(E)\right)\right] .
\end{aligned}
$$

В зависимости от определения подмножества каналов $\alpha$ формула (18) будет описывать либо полные, либо парциальные характеристики возбуждения квазистационарных состояний атомов.

Из выражений (15), (18) видно, что возбуждение и распад $m$-го квазистационарного состояния по группе каналов $\alpha \in \Delta$ характеризуется двумя комплексными функциями $N_{\alpha m}(E)$ и $\eta_{m}(E)=E_{m}(E)-i \Gamma_{m}(E) / 2$. Функции $\eta_{m}(E)$ входят в выражение (11). Нули этих функций на комплексной плоскости энергии определяют полюса $S$-матрицы рассеивания, которые соответствуют возбуждению квазистационарных состояний. Согласно положений работы Зигерта [26], вещественная часть комплексной энергии полюса определяет положение резонанса, а мнимая часть - его ширину. Таким образом, задача определения положений и ширин резонансов состоит в нахождении решений системы несвязанных комплексных уравнений

$$
E-E_{m}(E)+i \tilde{A}_{m}(E) / 2=0 .
$$

Равенство (19) может выполняться только при комплексных значениях энергии $E$. Решение уравнения (19) требует аналитического продолжения в задаче нахождения собственных векторов и собственных значений комплексной матрицы $W_{n m}(E)$ :

$$
W_{n m}(E)=E_{n} \delta_{n m}+F_{n m}(E)-i \gamma_{n m}(E) .
$$

Так как элементы матрицы $W_{n m}(E)$ являются функциями полной энергии $E$, то ее собственные значения и собственные векторы тоже будут зависеть от $E$. Поскольку мнимая и вещественная части этой матрицы связаны между собой преобразованием Гильберта, то аналитическим продолжением $W_{n m}(E)$ на всю комплексную плоскость энергии $E=E_{1}+i E_{2}$ будет матрица вида

$$
W_{n m}^{z}(E)=E_{n} \delta_{n m}+\int_{-\infty}^{\infty} \frac{\gamma_{n m}\left(E^{\prime}\right)}{E_{1}+i E_{2}-E^{\prime}} d E^{\prime}
$$

Используя соотношения для контурного интеграла типа Коши, можно показать, что

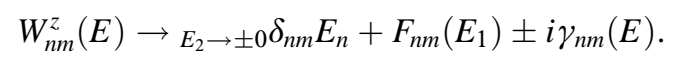

Положения полюсов определяются значениями $E_{1}$ и $E_{2}$, при которых матрица $\delta_{n m}\left(E_{1}+i E_{2}\right)-W_{n m}^{z}\left(E_{1}+i E_{2}\right)$ вырождается. Условием вырождения матрицы является равенство нулю ее детерминанта, откуда имеем систему уравнений для определения комплексных энергий квазистационарных состояний

$$
\left\{\begin{array}{l}
\operatorname{Re}\left[\operatorname{det}\left\|\left(E_{1}+i E_{2}\right) \delta_{n m}-W_{n m}^{z}\left(E_{1}+i E_{2}\right)\right\|\right]=0, \\
\operatorname{Im}\left[\operatorname{det}\left\|\left(E_{1}+i E_{2}\right) \delta_{n m}-W_{n m}^{z}\left(E_{1}+i E_{2}\right)\right\|\right]=0,
\end{array}\right.
$$

Таким образом, чтобы определить положение и ширину m-резонанса, необходимо решить систему уравнений (23). Как было показано выше, введение системы спектроскопических параметров, характеризующих профили АИС в сечениях ионизации, связано с возможностью использования резонансного приближения в формулах (11)-(18). В случае, когда это приближение можно применить, положения и ширины резонансных состояний определяются из (23) в первом порядке теории возмущений.

Найдем решения следующих уравнений:

$$
E_{m}\left(\tilde{E}_{m}\right)-\tilde{E}_{m}=0 .
$$

Корни этих уравнений будем считать положениями АИС, а ширины определим как значения функций $\Gamma_{m}(E)$ при $E=\tilde{E}_{m}$. В задачах расчета дифференциальных характеристик возбуждения АИС часто бывает необходимо определить парциальные ширины распада квазистационарных состояний в несколько каналов распада. В диагонализационном приближении парциальная ширина вводится через матричный элемент распада следующим образом:

$$
\tilde{\Gamma}_{m}(E)=2 \pi \sum_{\lambda}|\langle m|\hat{V}| \lambda \varepsilon\rangle|^{2}, \quad \lambda \in \alpha .
$$

Полная ширина соответствует $\alpha=\Delta\left(\tilde{\Gamma}_{m}(E)\right)=\Gamma_{m \Delta}(E)$. $\mathrm{B}$ случае взаимодействующих квазистационарных состояний парциальные ширины вводим по аналогии с 
диагонализационным приближением (25), а именно

$$
\tilde{\Gamma}_{j}(E)=2 \pi \sum_{\lambda} \tilde{V}_{m \alpha}(E) \tilde{V}_{m \lambda}^{*}(E)
$$

где $j$ - индекс парциального канала. Однако в даном случае полная ширина $\Gamma_{m}(E)$, определенная диагонализацией комплексной матрицы, не совпадает с суммой парциальных ширин, как это было в случае (25).

В практических расчетах методом сильной связи каналов, например [27], положение резонанса определяется значением энергии, при котором собственная фаза соответствующего канала равна $\pi / 2$. Определение этой величины сводится к решению следующих уравнений:

$$
\operatorname{Arg}\left[\eta_{i}(E)\right]=\pi / 2
$$

где $\eta_{i}(E)$ - собственное число $S$-матрицы, соответствующее каналу $i$. Набор ширин может быть определен также и через значение функции $\Gamma_{m}(E)$ в точках $\tilde{E}_{m}$, которые удовлетворяют (24). Однако такой способ определения положений и ширин имеет существенный недостаток - множество решений уравнений (24) содержит также корни, обусловленные присутствием в непрерывном спектре атома так называемых „резонансов формы“. Решение вопроса об идентификации АИС в данном случае состоит в непосредственном анализе полюсов $S$-матрицы рассеяния. При таком способе определения положений резонансных состояний каждый канал реакции имеет свой набор решений соответствующего уравнения [6,27]. Что касается ширин, то при использовании аппарата метода сильной связи каналов они определяются фитированием функций $\eta_{i}(E)[6]$.

Состояния гелия в области непрерывного спектра, где находятся АИС, сходящиеся к третьему порогу, описывались волновой функцией [24], которая учитывает все межконфигурационные взаимодействия конечного числа базисных конфигураций, соответствующие двухэлектронным возбуждениям в области между вторым и третьим порогами (закрытые каналы), и электрона с позитивным значением энергии над основным и первым возбужденным состояниями иона $\mathrm{He}^{+}$(открытые каналы). В расчетах учитывались состояния с полным моментом атома гелия $L \leq 3$.

Для каждого момента $L$ подпространство закрытых каналов заполнялось двадцатью конфигурациями, а в качестве базисных функций для их описания использовались кулоновские волновые функции с зарядом $z=2$. Затем подпространство этих состояний предварительно диагонализировалось. Подпространство открытых каналов включало три конфигурации для $L=0$ и четыре для других моментов $L$. Это соответствует включению в расчет каналов, соответствующих основному и первому возбужденному состоянию иона $\mathrm{He}^{+} 1 s \varepsilon L, 2 s \varepsilon L$, $2 p \varepsilon(L-1), 2 p \varepsilon(L+1)$.

\section{Результаты расчетов}

Для описания основного состояния атома Не один из соавторов настоящей работы и коллеги в работах $[24,25]$ использовали 41-параметрическую волновую функцию Твида [20] и получили точные значения параметров АИС атома гелия, находящиеся в области выше порога образования возбужденных ионов. Представляет интерес

Таблица 1. Парциальные ширины нижних 5-и ${ }^{1} S$-резонансов, полученных в диагонализационном приближении в представлении вещественных чисел

\begin{tabular}{c|c|c|c|c|c}
\hline № & $E, \mathrm{eV}$ & $\Gamma, \mathrm{eV}$ & $1 s \varepsilon s, \mathrm{eV}$ & $2 s \varepsilon s, \mathrm{eV}$ & $2 p \varepsilon p, \mathrm{eV}$ \\
\hline 1 & 69.39400 & 0.08235 & 0.00264 & 0.07904 & 0.00067 \\
2 & 70.48503 & 0.17282 & 0.00659 & 0.04469 & 0.12154 \\
3 & 71.40519 & 0.04091 & 0.00269 & 0.03755 & 0.00067 \\
4 & 71.91078 & 0.04045 & 0.00226 & 0.01187 & 0.02632 \\
5 & 72.07814 & 0.01973 & 0.00155 & 0.01767 & 0.00053
\end{tabular}

Таблица 2. Парциальные ширины нижних 5-и ${ }^{1} P$-резонансов, полученных в диагонализационном приближении в представлении вещественных чисел

\begin{tabular}{c|c|c|c|c|c|c}
\hline № & $E, \mathrm{eV}$ & $\Gamma, \mathrm{eV}$ & $1 s \varepsilon p, \mathrm{eV}$ & $2 s \varepsilon p, \mathrm{eV}$ & $2 p \varepsilon s, \mathrm{eV}$ & $2 p \varepsilon d, \mathrm{eV}$ \\
\hline 1 & 69.91937 & 0.16584 & 0.00033 & 0.03206 & 0.09450 & 0.03899 \\
2 & 71.24768 & 0.00101 & 0.00001 & 0.00063 & 0.00027 & 0.00011 \\
3 & 71.47437 & 0.06436 & 0.00011 & 0.00240 & 0.00368 & 0.05817 \\
4 & 71.66483 & 0.06522 & 0.00033 & 0.01596 & 0.04076 & 0.00818 \\
5 & 71.78036 & 0.00066 & 0.00001 & 0.00007 & 0.00003 & 0.00057
\end{tabular}

Таблица 3. Парциальные ширины нижних 5-и ${ }^{1} D$-резонансов, полученных в диагонализационном приближении в представлении вещественных чисел

\begin{tabular}{c|c|c|c|c|c|c}
\hline № & $E, \mathrm{eV}$ & $\Gamma, \mathrm{eV}$ & $1 s \varepsilon d, \mathrm{eV}$ & $2 s \varepsilon d, \mathrm{eV}$ & $2 p \varepsilon p, \mathrm{eV}$ & $2 p \varepsilon f, \mathrm{eV}$ \\
\hline 1 & 69.66939 & 0.15198 & 0.00001 & 0.00342 & 0.14499 & 0.00356 \\
2 & 70.50481 & 0.12298 & 0.00076 & 0.00837 & 0.11141 & 0.00244 \\
3 & 71.22368 & 0.01108 & 0.00227 & 0.00306 & 0.00332 & 0.00244 \\
4 & 71.54640 & 0.21438 & 0.00571 & 0.00076 & 0.20598 & 0.00193 \\
5 & 71.56122 & 0.03308 & 0.00018 & 0.00776 & 0.02507 & 0.00008
\end{tabular}

Таблица 4. Парциальные ширины нижних 5-и ${ }^{1} P$-резонансов, полученных в диагонализационном приближении в представлении вещественных чисел

\begin{tabular}{c|c|c|l|c|c|c}
\hline № & $E, \mathrm{eV}$ & $\Gamma, \mathrm{eV}$ & $1 s \varepsilon f, \mathrm{eV}$ & $2 s \varepsilon f, \mathrm{eV}$ & $2 p \varepsilon d, \mathrm{eV}$ & $2 p \varepsilon g, \mathrm{eV}$ \\
\hline 1 & 70.88120 & 0.08686 & 0.00006 & 0.03679 & 0.02921 & 0.02079 \\
2 & 71.48071 & 0.00495 & 0.0000001 & 0.00185 & 0.00244 & 0.00065 \\
3 & 71.99411 & 0.02403 & 0.00002 & 0.00749 & 0.01043 & 0.00608 \\
4 & 72.13817 & 0.00074 & 0.0000004 & 0.00054 & 0.00016 & 0.00004 \\
5 & 72.13959 & 0.00347 & 0.000002 & 0.00160 & 0.00128 & 0.00058
\end{tabular}


Таблица 5. Параметры нижнего ${ }^{1}$ Р-автоионизационного состояния атома Не в области энергий выше порога образования возбужденных ионов, применение разных волновых функций основного состояния

\begin{tabular}{l|c|c|c|c|c|c}
\hline Функции & $E, \mathrm{eV}$ & $\Gamma, \mathrm{eV}$ & 1 ses, $\mathrm{eV}$ & $2 s e p, \mathrm{eV}$ & $2 p e p, \mathrm{eV}$ & $2 p e d, \mathrm{eV}$ \\
\hline $\begin{array}{l}\text { 6-параметрическая } \\
\text { функция Хиллерааса [28] }\end{array}$ & 69.89 & 0.150 & $0.893(-3)$ & $0.918(-1)$ & $0.313(-1)$ & $0.257(-1)$ \\
\hline $\begin{array}{l}\text { 41-параметрическая } \\
\text { функция Твида }\end{array}$ & 69.92 & 0.165 & $0.312(-3)$ & $0.945(-1)$ & $0.320(-1)$ & $0.389(-1)$ \\
\hline $\begin{array}{l}\text { 6-параметрическая } \\
\text { функция Хиллерааса }\end{array}$ & 69.90 & 0.154 & $0.871(-3)$ & $0.814(-1)$ & $0.315(-1)$ & $0.407(-1)$ \\
\hline $\begin{array}{l}\text { 8-параметрическая } \\
\text { функция Хиллерааса }\end{array}$ & 69.81 & 0.158 & $0.852(-3)$ & $0.836(-1)$ & $0.310(-1)$ & $0.425(-1)$ \\
\hline $\begin{array}{l}\text { Функция } \\
\text { Монте-Карло [21] }\end{array}$ & 69.91 & 0.159 & $0.476(-3)$ & $0.991(-1)$ & $0.235(-1)$ & $0.359(-1)$
\end{tabular}

анализ влияния выбора функции основного состояния на парциальные характеристики АИС. Расчет парциальных ширин АИС в задаче фотоионизации гелия проведен в работе [28] на основе применения 6-параметрической функции Хиллерааса в диагонализационном приближении. В работах $[24,25]$ наряду с диагонализационным приближением использовался метод взаимодействующих конфигураций в представлении комплексных чисел, и результаты приведены во всех приближениях, которые из него следуют, однако во всех вариантах расчетов в качестве функции основного состояния атома гелия применялась 41-параметрическая функция Твида [20]. С другой стороны, смысл понятию парциальных ширин можно придать только в диагонализационном приближении, поэтому ниже мы также приводим расчеты в диагонализационном приближении [22-25]. В частности, в табл. 1-4 приводим полученные парциальные ширины нижних АИС.

Анализ применения разных волновых функций выполнен на примере нижнего ${ }^{1} P$-состояния, описанного в табл. 2. Этот анализ представлен в табл. 5.

В табл. 5 приведены параметры нижнего ${ }^{1} P$-состояния в задаче фотоионизации атома гелия выше порога образования возбужденных ионов. Расчеты выполнены для разных волновых функций основного состояния. Анализ показывает, что параметры квазистационарных состояний зависят от выбора волновой функции основного состояния.

\section{Выводы}

Расчеты резонансных сечений в задачах фотоионизации и анализ резонансных профилей являются источником сведений о структуре атомных систем и позволяют более адекватно отобрать теоретические модели, а выбор волновой функции основного состояния влияет на значение соответствующих параметров резонансов.

Исследования в области энергий возбуждения выше второго порога ионизации, или выше порога образова- ния возбужденных ионов $\mathrm{He}^{+}$, во многих аспектах похожи на более ранние исследования, которые проводились в области энергий между первым и вторым порогами ионизации, но в этих исследованиях открываются значительно более широкие возможности. Более богатым является спектр рассматриваемых характеристик. Это определяется возможностью заселения в процессах прямой, а также резонансной ионизации фотонами и электронами как основного, так и возбужденного состояний остаточных ионов, переходящих затем в основное состояние путем излучения фотона. Таким образом, открывается возможность исследования профилей резонансов, сходящихся к порогу $N=3$ атома Не как в полных, так и в парциальных сечениях ионизации, а учет связи каналов в этих процессах является обязательным. Выбор волновой функции основного состояния прямо влияет на значения соответствующих матричных элементов, входящих в сечения ионизации.

Результаты расчетов демонстрируют, что в отличие от полных ширин АИС парциальные ширины являются существенно разными для разных волновых функций основного состояния. Из табл. 5 видно, что полные ширины также отличаются друг от друга, когда они рассчитаны на основе разных волновых функций в рамках одного и того же подхода, однако парциальные ширины при этом отличаются между собой сильнее. Это можно попытаться объяснить отличием друг от друга волновых функций основного состояния. Однако для детального изложения причин данных фактов необходимы дальнейшие исследования.

\section{Список литературы}

[1] Burke P.G. // Effects of configuration interaction on electron and photon interactions with atoms. In: Electron and photon interactions with atoms. NY.: Plenum Press, 1976. P. 1-25.

[2] Burke P.G., Berrington K.A., Sukumar C.V. // J. Phys. B: At. Mol. Phys. 1981. Vol. 14. N 2. P. 289-306.

[3] Luke T.M. // J. Phys. B: At. Mol. Phys. 1975. Vol. 8. N 9. P. 1501-1506. 
[4] Fano U. // Phys. Rev. 1961. Vol. 124. N 6. P. 1866-1878.

[5] Fano U., Cooper J.W. // Rev. Mod. Phys. 1968. Vol. 40. N 3. P. 441-507.

[6] Жигунов В.П., Захарьев Б.Н. // Методы сильной связи каналов в квантовой теории рассеяния. М.: Атомиздат, 1974. Р. 224.

[7] Гайлитис М.К. // УФН. 1975. Т. 116. Вып. 4. С. 665-686.

[8] Froese Fischer $C$. // The Hartree-Fock method for atoms a numerical approach. NY.: John Wiley and Sons Inc., 1977. $320 \mathrm{p}$.

[9] Froese Fischer C. // Comput. Phys. Commun. 1978. Vol. 14. $\mathrm{N} 1-2$. P. $145-153$.

[10] Hylleraas E.A. // Z. Phys. 1928. Bd. 48. N 7-8. P. 469-494.

[11] Hylleraas E.A. // Z. Phys. 1929. Bd. 54. N 5-6. P. 347-366.

[12] Hylleraas E.A. // Z. Phys. 1930. Bd. 65. N 3-4. P. 209-225.

[13] Решетняк В.И., Федоров Ф.И. // ДАН СССР. 1982. Т. 263. N 6. C. $1356-1359$.

[14] Фок В.А. // Изв. АН СССР. Сер. Физ. 1954. Т. 18. N 2. C. $161-169$.

[15] Froese Fischer C. // Comput. Phys. Commun. 2000. Vol. 128. N 3. P. $635-636$.

[16] Pekeris C.L. // Phys. Rev. 1959. Vol. 115. N 5. P. 1216-1221.

[17] Pekeris C.L. // Phys. Rev. 1962. Vol. 127. N 2. P. 509-511.

[18] Головинский П.А., Киян И.Ю. // УФН. 1990. Т. 160. Вып. 6. C. $97-140$.

[19] Евсеев М.К., Матвеев В.И. // ЖТФ. 2008. Т. 78. Вып. 8. C. $28-33$.

[20] Tweed R.J. // J. Phys. B: At. Mol. Phys. 1972. Vol. 5. N 4. P. 810-819.

[21] Alexander S.A., Coldwell R.L. // Int. J. Quant. Chem. 1997. Vol. 63. N 5. P. 1001-1022.

[22] Balashov V.V., Grishanova S.I., Kruglova I.M., Senashenko V.S. // Phys. Lett. A. 1968. Vol. 27. N 2. P. 101-102.

[23] Балашов В.В., Липовецкий С.С., Сенаменко В.С. // ЖЭТФ. 1972. Т. 63. Вып. 2. С. 1622-1627.

[24] Burkov S.M., Letyaev N.A., Strakhova S.I., Zajac T.M. // J. Phys. B: At. Mol. Opt. Phys. 1988. Vol. 21. N 7. P. 1195-1208.

[25] Burkov S.M., Strakhova S.I., Zajac T.M. // J. Phys. B: At. Mol. Opt. Phys. 1990. Vol. 23. N 20. P. 3677-3690.

[26] Siegert A.J. // Phys. Rev. 1939. Vol. 56. N 8. P. 750-752.

[27] Seaton M.I. // J. Phys. B: At. Mol. Phys. 1974. Vol. 7. N 14. P. $1817-1840$.

[28] Senashenko V.S., Wague A. // J. Phys. B: At. Mol. Phys. 1979. Vol. 12. N 8. P. L269-L273. 